University of Nebraska - Lincoln

DigitalCommons@University of Nebraska - Lincoln

8-15-2006

\title{
Load Transfer Issues in the Tensile and Compressive Behavior of Multiwall Carbon Nanotubes
}

G. A. Shen

Florida State University

S. Namilae

FAMU-FSU College of Engineering, Florida State University, Tallahassee, FL

Namas Chandra

University of Nebraska-Lincoln, nchandra2@unl.edu

Follow this and additional works at: https://digitalcommons.unl.edu/mechengfacpub

Part of the Mechanical Engineering Commons

Shen, G. A.; Namilae, S.; and Chandra, Namas, "Load Transfer Issues in the Tensile and Compressive Behavior of Multiwall Carbon Nanotubes" (2006). Mechanical \& Materials Engineering Faculty Publications. 26.

https://digitalcommons.unl.edu/mechengfacpub/26

This Article is brought to you for free and open access by the Mechanical \& Materials Engineering, Department of at DigitalCommons@University of Nebraska - Lincoln. It has been accepted for inclusion in Mechanical \& Materials Engineering Faculty Publications by an authorized administrator of DigitalCommons@University of Nebraska Lincoln. 
Published in Materials Science and Engineering: A 429:1-2 (August 15, 2006), pp. 66-73; doi: 10.1016/j.msea.2006.04.110

Copyright $\odot 2006$ Elsevier B.V. Used by permission. http://www.elsevier.com/locate/msea

Submitted September 9, 2005; revised April 14, 2006; accepted April 24, 2006; published online June 27, 2006.

\title{
Load transfer issues in the tensile and compressive behavior of multiwall carbon nanotubes
}

\author{
G. A. Shen, S. Namilae, and N. Chandra \\ Department of Mechanical Engineering, FAMU-FSU College of Engineering, \\ Florida State University, Tallahassee, FL 32310, U.S.A. \\ Corresponding author - N. Chandra
}

\begin{abstract}
Carbon nanotubes (CNT) are considered to be ultra strong and stiff reinforcements for structural composite applications. The load transfer between the inner and outer nanotubes in multiwall carbon nanotubes (MWCNT) has to be clearly understood to realize their potential in not only composites, but also other applications such as nano-springs and nano-bearings. In this paper, we study the load transfer between the walls of multiwall nanotubes both in tension and compression using molecular dynamics simulations. It is found that very minimal load is transferred to the inner nanotube during tension. The load transfer in compression of capped nanotubes is much greater than that in tension. In the case of uncapped nanotubes, the inner nanotube is deformed in bending, only after the outer nanotube is extensively deformed by buckling. It is found that the presence of a few interstitial atoms between the walls of multiwall nanotube can improve the stiffness and enhance the load transfer to the inner nanotubes both in tension and compression.
\end{abstract}

Keywords: multiwall carbon nanotubes, load transfer, molecular dynamics, nanocomposites

\section{Introduction}

The combination of high strength, stiffness and aspect ratio makes carbon nanotubes ideal reinforcements for ultra high strength composites. Stiffness of CNTs measured experimentally [1] and calculated from simulations [2] is of the order of $1000 \mathrm{GPa}$, while the nearest competitive fiber ( $\mathrm{SiC}$ whiskers) has utmost $400 \mathrm{GPa}$ in stiffness [3]. CNTs have tensile strength of up to $150 \mathrm{GPa}$ [4] and can absorb large quantities of energy during elastic and inelastic deformations [5, 6]. This combination of mechanical properties has raised the possibility of obtaining super-strong and stiff composites with CNTs as reinforcements. Further, the excellent electrical and optical properties of CNTs facilitate development of multifunctional products [7]. Numerous researchers have fabricated composites in bulk and thin film forms with different matrix materials [8].

It is well known that multiwall carbon nanotubes are more commonly obtained during the synthesis of carbon nanotubes by various existing methods such as arch discharge and chemical vapor deposition [9]. Because of this, structural and thermal applications of carbon nanotubes in all likelihood will involve multiwall nanotubes, at least in the foreseeable future. In composite materials, the load trans- fer between fiber and matrix plays a key role in mechanical properties such as strength, stiffness and fracture resistance [10]; when MWCNTs are used as fibers an additional complication exists due to the load transfer between the outer and inner nanotubes. It is essential to understand the inter-tube load transfer mechanisms between nanotubes for optimizing the use of MWCNTs (volume fraction and distribution) in composites and other applications. The inter-tube load transfer is also expected to play a key role in other nano-mechanical systems such as nano-springs, bearings which are based on "sword-in sheath" mechanism of inter-tube separation [11-13].

There have been extensive studies on the mechanics of carbon nanotubes but relatively few researchers have studied the load transfer between the walls of multiwall nanotubes. Cumings and Zettl demonstrated extension of inner nanotube out of a multiwall nanotube inside a transmission electron microscopy. Akita and Nakayama [14] have also experimentally extracted the inner shell of nanotube using electrical means. The inter-tube interactions in above studies have been found to be consistent with theoretical models based on van der Waals interaction. Schaddler et al. [15] report that compressive modulus of nanotube composite is higher than that in tension; they explain this on the basis of 
higher inter-tube load transfer in compression. Yu and coworkers [16] have performed tensile tests on individual multiwall nanotubes; they report that failure occurs only in the outer nanotube indicating that there is very limited load transfer between the inner and outer nanotubes. Ru [17] has recently studied the problem of buckling of a double walled carbon nanotube using continuum analysis. Xia and Curtin [18] have studied the frictional interaction between nanotubes using simulations of pullout tests. In spite of these studies there is no clear understanding as to how the interwall load transfer can affect the overall tensile and compressive behavior of the multiwall nanotube. Molecular dynamics simulations can provide atomic detail absent in continuum analysis and hence can provide valuable insights into the behavior of these nanoscale systems.

Load transfer between fiber (multiwall nanotube) and matrix in different loading conditions is a crucial issue in developing high strength composites. If the load transfer is not effective, then the useful length of fiber partaking in loading reduces decreasing the stiffness enhancement. There are conflicting observations regarding load transfer and interfacial strength in CNT composites; however, functionalization (surface chemical modification of carbon nanotubes) is expected to improve the interface properties of CNT composites. Namilae and Chandra have earlier used molecular dynamics simulations and multiscale model to show that the chemical attachments between polymer matrix and nanotube fiber can enhance mechanical properties of the composite $[10,19]$. Several researchers report that irradiation of carbon nanotubes can cause chemical attachments between the walls of nanotubes [20, 21]; the effect of these chemical attachments on the load transfer between the walls of multiwall nanotube is not clearly understood and is one of the issues addressed in this paper.

The objective of this paper is to study the effect of nonbonded interactions between the walls of MWCNTs on their mechanical properties. We use molecular dynamics simulations to study the mechanical response of capped and uncapped (or fractured) nanotubes in tension and compression. Another objective of the paper is to study the difference in the mechanics of deformation when the MWCNTs are subject to tension and compression. One of the ways to improve the load transfer in multiwall nanotubes is by introducing chemical bonding between the walls of nanotubes. We have studied the effect of chemical attachments between the walls of nanotube on the mechanical properties of multiwall nanotubes.

\section{Inter-nanotube interaction in shear and tension}

We primarily employ molecular dynamics and statics simulations in the present study. Tersoff-Brenner bond-order potential which has coordination dependant terms that enable modeling bond conjugations in carbon is used for $\mathrm{C}-\mathrm{C}$ and $\mathrm{C}-\mathrm{H}$ interactions $[22,23]$. This potential has been used by several investigators to study deformation of carbon nanotubes and is a widely used and appropriate potential $[2,6,10,18,19,24,32]$. The interactions between the walls of nanotubes are based on long range van der Waals forces. These non-bonded interactions are modeled using 12-6 Lennard-Jones potential given by the Equation (1) with

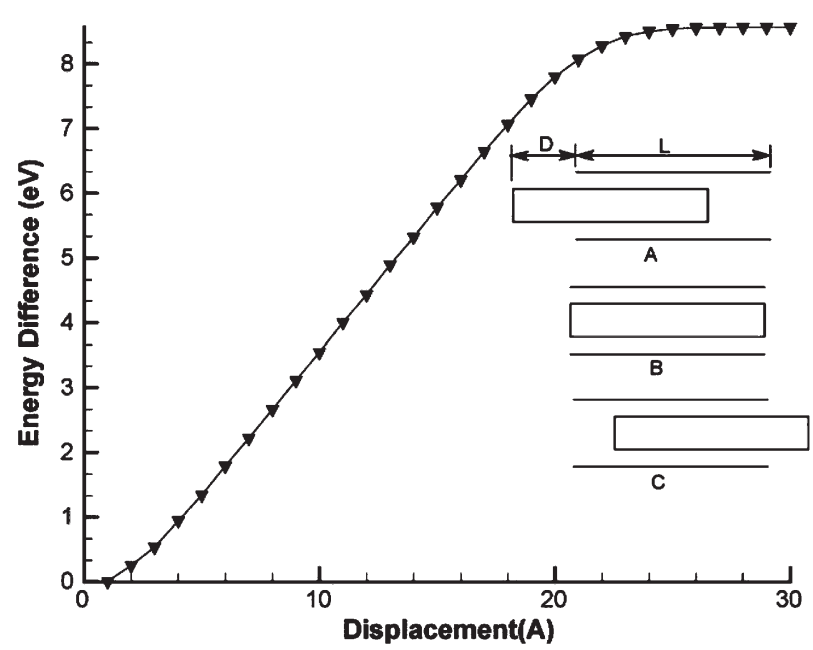

Figure 1. Energy vs. displacement for pullout of inner nanotube for $(15,0),(6,0)$ multiwall carbon nanotube. Inset shows the schematic of the pullout test.

$\varepsilon=2.86 \mathrm{meV}$ and $\sigma=3.4 \mathrm{~A}$. Here $r$ is the distance between atoms, and $V$ is potential energy.

$$
V(r)=4 \varepsilon\left(\left(\frac{\sigma}{r}\right)^{12}-\left(\frac{\sigma}{r}\right)^{6}\right)
$$

We first study the energetics of carbon nanotubes by performing molecular statics simulations of pullout of inner nanotube from a double walled CNT. The interaction between the inner and outer nanotubes of $(6,0),(15,0)$ double walled nanotube is examined by pulling the inner nanotube while the outer nanotube is left intact. Energetically stable intermediate states are obtained by sequentially displacing the inner nanotube followed by energy minimization. The change in energy versus displacement plot is as shown in Figure 1 . The energy value reaches a plateau when the inner $(6,0)$ nanotube no longer interacts with the outer $(15,0)$ nanotube. The specific displacement value depends on the length of the nanotubes as shown. An estimate of interfacial shear stress can be obtained as the differential of energy difference $\tau_{\text {int }}=\left(1 / A_{\mathrm{CNT}}\right)(\partial(\Delta E) /(\partial r))$. Here $A_{C N T}$ is the surface area of CNT and $r$ is displacement of inner nanotube. Based on our calculation we obtain interface strength of $54.7 \mathrm{MPa}$ for $(6,0)(15,0)$ double walled nanotube. The value of interfacial energy increases with increasing diameter of nanotube as the interaction area increases; however, the order of the magnitude of energy and interfacial shear stress remain the same. For example the interfacial stress for $(10,10)$ nanotube embedded in a $(15,15)$ nanotube is $59.4 \mathrm{MPa}$. This interfacial energy and strength are very low since they are based on non-bonded van der Waal's interactions.

We will now explore tensile and compressive behavior of capped and uncapped multiwall carbon nanotubes concurrently with local stress measures to study the load transfer between the walls of nanotube under both tension and compression. We invoke the use of local stress measures in each of the tubes to study the load transfer between the tubes. The definition of stress in a discreet atomic setting depends on the definition of homogeneity of that stress measure. These 


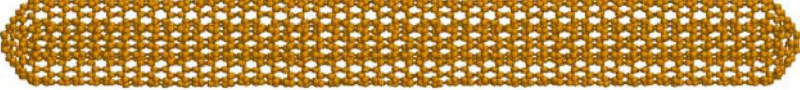

(a)

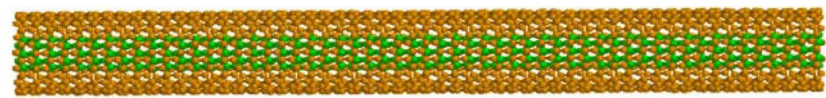

(b)

Figure 2. Schematic of carbon nanotubes studied in this work (a) $(6,0)$ $(15,0)$ capped double-wall carbon nanotube and $(\mathbf{b})(6,0)(15,0)$ uncapped double-wall carbon nanotube.

concepts are discussed in detail elsewhere [24]; however they are mentioned here briefly for the sake of completeness. Stress at a point, in the sense of continuum mechanics implicitly assumes that a homogenous state of stress exists within the appropriately chosen infinitesimal volume surrounding that point. When this concept is extended to discrete lattice mechanics, we need to identify a volume around a given point over which the stress becomes homogeneous. Various definitions of atomic stress such as virial stress [25], BDT stress [26] and Lutsko stress [27, 28] can be used for different volumes of homogeneity. We primarily use Lutsko stress $\sigma_{i j}^{\text {Lutsko }}$ to characterize stress. It can be used when the volume in consideration is lesser than the total volume of the system. It is given by:

$$
\sigma_{i j}^{\text {Lutsko }}=\frac{1}{\Omega^{\text {Avg }}} \sum_{\alpha=1, n}\left(\frac{1}{2} m^{\alpha} v_{i}^{\alpha} v_{j}^{\alpha}+\sum_{\beta=1, n} r_{\alpha \beta}^{j} f_{\alpha \beta}^{i} l_{\alpha \beta}\right)
$$

Here $\Omega^{\mathrm{Avg}}$ is the averaging volume, $v, r, f$ the velocity force and radial vectors, $m$ the atom mass, $a$ and $\beta$ the atomic indices, and $i$ and $j$ are the indices of the stress tensor. $l_{a \beta}$ denotes the fraction of $a-\beta$ bond lying inside the averaging volume. We calculate the local volume $\Omega^{A v g}$ as the cylindrical volume of individual nanotube concerned with a thickness of 3.4 A. It is interesting to note that when the averaging volume is equal to the total volume of the system; the definition of Lutsko stress equals that of virial stress given by:

$$
\sigma_{i j}^{\text {virial }}=\frac{1}{\Omega^{\text {Total }}} \sum_{\alpha=1, n}\left(\frac{1}{2} m^{\alpha} v_{i}^{\alpha} v_{j}^{\alpha}+\sum_{\beta=1, n} r_{\alpha \beta}^{j} f_{\alpha \beta}^{i}\right)
$$

Here $\Omega^{\text {Total }}$ is the total volume of the nanotube.

Tensile and compressive loading is applied on two sets of multiwall nanotubes; capped and uncapped $(6,0)$ and $(15,0)$ as well as uncapped $(10,10)(15,15)$ nanotubes (see Figure 2 ). In order to apply the deformation, few atoms at both the ends of nanotubes are held fixed and displaced (by $0.05 \mathrm{~A}$ ) outward or inward (for tension and compression, respectively) followed by equilibration (for 1500 time steps). To study the load transfer between the inner and outer nanotubes, we compare the cases when the loading is applied on both nanotubes and outer nanotube alone. Loading both the nanotubes corresponds to an ideal scenario, where there is perfect bonding between the inner and outer nanotubes.

Figure 3 shows the stress-strain plots for tensile loading of $(6,0)(15,0)$ capped nanotube. Plot (a) of Figure 3 is

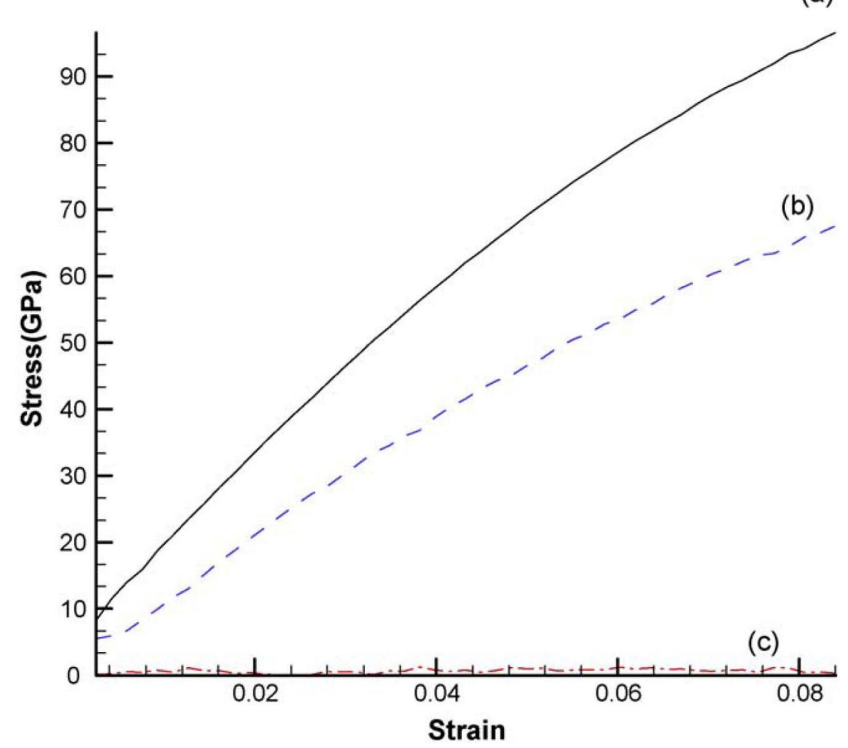

Figure 3. Stress-strain response of $(15,0)(6,0)$ capped multiwall nanotube. (a) Virial stress when both tubes are loaded, (b) virial stress when only outer nanotube is loaded and (c) Lutsko stress in inner nanotube is much lesser than that in the outer nanotube.

the virial stress versus applied strain when both inner and outer nanotubes are subject to tensile loading. Plot (b) shows the virial stress versus strain when the loading (displacement) is applied on the outer nanotube alone. It can be noted that the Young's modulus (calculated at zero strain) is much higher when both the nanotubes are loaded simultaneously. It is $888 \mathrm{GPa}$ when the outer nanotube alone is loaded compared to $1185 \mathrm{GPa}$ when both the nanotubes are loaded. Plot (c) shows the Lutsko stress for the inner tube when the outer tube alone is loaded. It can be observed that the stress experienced by the inner nanotube is very less compared to the stress experienced by the outer nanotube. This reemphasizes the fact that weak van der Waals bonds that exist between the walls of inner and outer nanotube do not effectively contribute to the load transfer, especially during tensile loading. These observations can be easily extended to multiwall nanotubes with more number of nanotubes. For example, even if there are six or seven nanotubes in a given MWCNT, if the outer nanotube alone is loaded only that tube resists the external load, consequently reducing the overall effectiveness of the multiwall nanotube.

Uncapped nanotubes can be considered as nanotubes that have fractured during loading. Figure 4 shows stress-strain plot for $(15,0)(6,0)$ nanotubes without end-caps. It can be observed from this figure that capped and uncapped nanotubes do not differ significantly in their tensile behavior. We obtain similar results for other multiwall nanotube systems such as $(10,10)(15,15)$ double wall nanotube.

\section{Inter nanotube interaction in compression}

Compressive response of carbon nanotubes is quite different from that of tension both for single-wall nanotubes and multiwall nanotubes. This difference can be attributed 


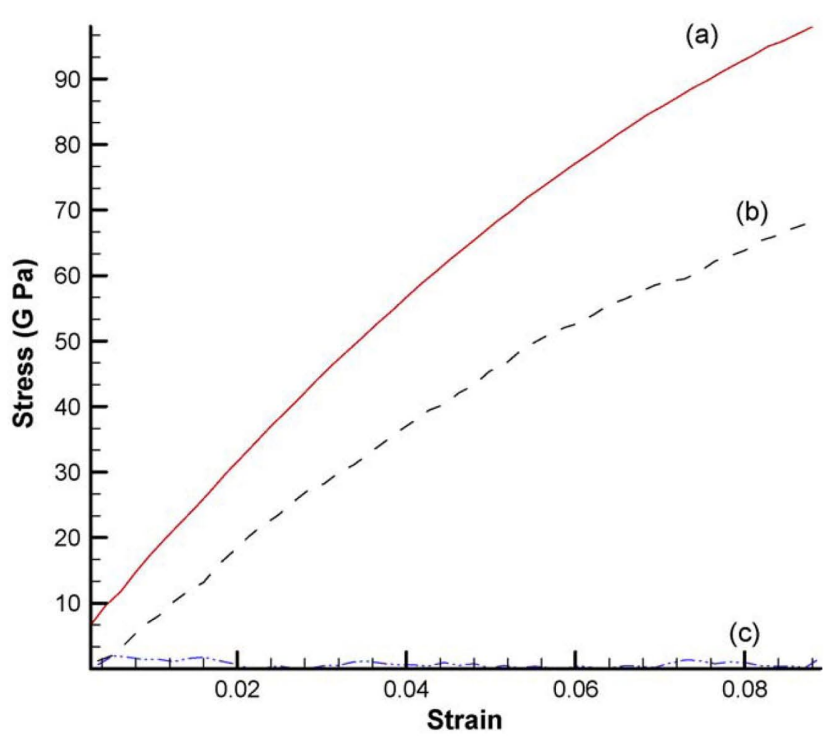

Figure 4. Stress-strain response of $(15,0)(6,0)$ uncapped multiwall nanotube. (a) Virial stress when both tubes are loaded, (b) virial stress when only outer nanotube is loaded and (c) Lutsko stress in inner nanotube is much lesser than that in the outer nanotube.

to two factors. Firstly at the bond level, there is asymmetry in tensile and compressive response of individual $\mathrm{C}-\mathrm{C}$ bond as manifest in the Tersoff-Brenner potential used in this work. Figure 5(a) shows the potential energy and force variation with respect to distance between two neighboring atoms in an infinite (periodic) graphene sheet (see Figure $5(b)$ ). The figure depicts this tension-compression asymmetry. It can be observed from the figure that the compressive deformation requires more energy than tensile deformation. Other potentials such as MM3 and Amber potentials which model C - C interactions also show similar behavior [29]. It should be noted that because of the anharmonicity of the potential the elastic response in compression and tension is different for each bond and hence for the overall structure composed of these bonds. This asymmetry is actually responsible for other properties such as a non-zero coefficient of thermal expansion.

The second reason for the asymmetry in tension and compression is due to the structural response. Because of the cy-

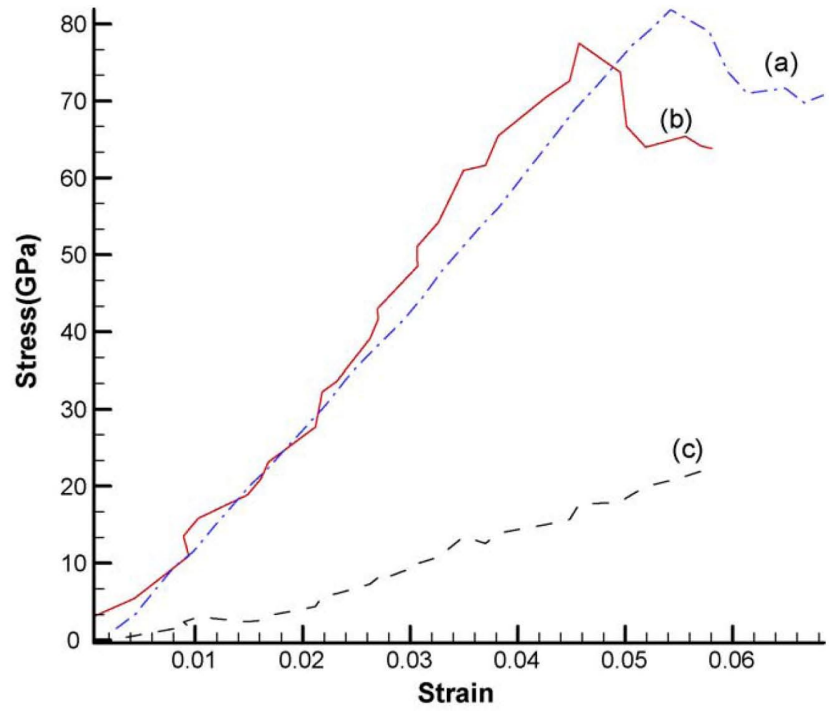

Figure 6. Compressive stress-strain response of $(15,0)(6,0)$ capped multiwall nanotube. (a) Virial stress when both tubes are loaded, (b) virial stress when only outer nanotube is loaded and (c) Lutsko stress in inner. Note that inner nanotube experiences considerable stress.

lindrical nature of the nanotubes, they have been observed to buckle when subject to compressive loading [30, 31]. In MWCNTs, there is an additional effect of inter-tube interactions. Also, unlike in tension, the difference between capped and uncapped nanotubes plays a key role in compression.

Figure 6 shows the compressive stress-strain plots for capped $(6,0)(15,0)$ double walled carbon nanotubes. The compressive loading is applied as described earlier using incremental displacements. Plot (a) in Figure 6 corresponds to the situation when atoms belonging to both inner and outer nanotubes are displaced inwards. For plot (b) the compressive loading was applied only on the atoms that belong to the outer nanotube. In both cases, viral stress of the double walled nanotube system is plotted against applied strain. Plot (c) shows the local stress experienced by the inner nanotube when the displacement is applied on the atoms of outer nanotube alone. The inflexion point in the stress-strain plot where the sudden drop in the stress is observed corresponds to the onset of infinitesimal buckling. We observe
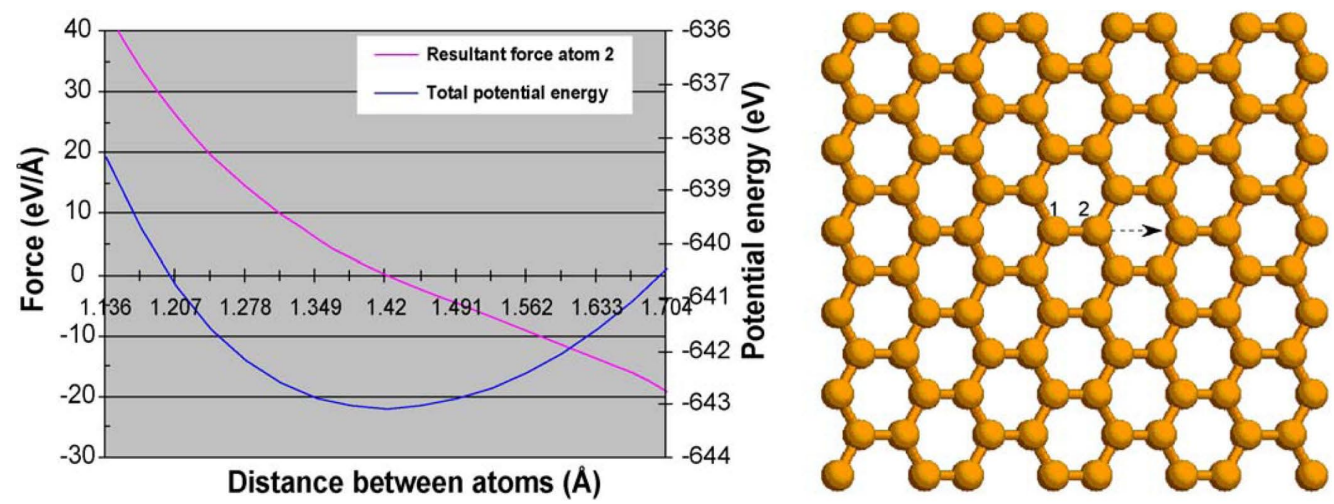

Figure 5. (a) Asymmetry in potential energy vs. displacement for Tersoff Brenner potential. (b) Schematic of graphene sheet distance between atoms 1 and 2 is considered in (a). 


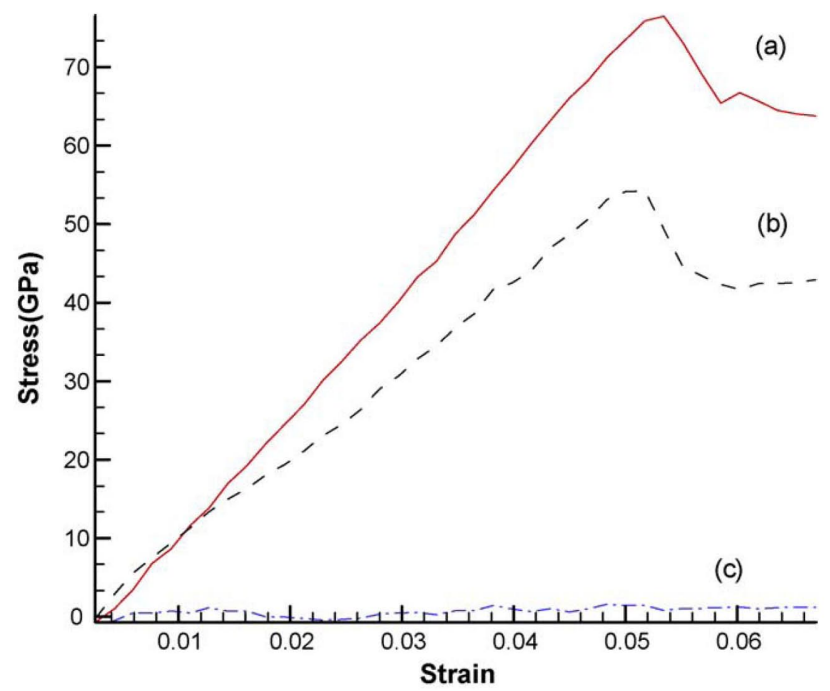

Figure 7. Compressive stress-strain response of $(15,0)(6,0)$ uncapped multiwall nanotube. (a) Virial stress when both tubes are loaded, (b) virial stress when only outer nanotube is loaded and (c) Lutsko stress in inner.

that in both the loading cases the buckling in the outer nanotube precedes that of the inner nanotube. The difference between plot (a) and plot (b) in Figure 6 is much lesser than corresponding stress-strain plots for tensile loading (Figure 4); in addition the stress experienced by the inner nanotube shown in plot (c) is much higher in compression than in tension (Figure 4 plot (c)). This unequivocally demonstrates that the load is transferred to inner nanotubes in compression at least when the nanotubes are capped.

We like to reiterate the difference in tensile and compressive behavior of MWCNTs. When tension is applied to the outer nanotube, the inner nanotube does not get loaded consequently the stiffness is low. On the other hand, the inner tube is loaded in compression even though only the outer nanotube is compressed. This tension compression asymmetry is driven by the buckled outer nanotube which comes in closer contact with the inner nanotube. Now we study the compressive deformation of uncapped nanotubes.

Figure 7 shows similar virial stress versus strain plots for $(6,0)(15,0)$ uncapped (or fractured) nanotube. Plot (a) shows virial stress versus strain when atoms of both nanotubes are displaced, while plot (b) corresponds to the case when displacements are applied only on the atoms of outer nanotube. Plot (c) shows the local stress experienced by the inner $(6,0)$ for this loading. A comparison between Figure 6 and Figure 7 shows that the load transfer in uncapped nanotubes is much lower than in capped nanotubes during initial stages of loading. This is reflected by the difference in plots (a) and (b) in Figure 7; also, the stress experienced by the inner nanotube (plot (c)) is minimal when the outer nanotube alone is loaded. We have observed similar results in other uncapped nanotube systems such as $(10,10)(15,15)$ double wall nanotubes.

It must be noted that the inner nanotube is deformed during the later stages of deformation. This deformation is a result of lateral forces (perpendicular to the nanotube length) exerted by the buckled outer nanotube. Figure 8 shows the snapshots of compressive deformation on $(6,0)$

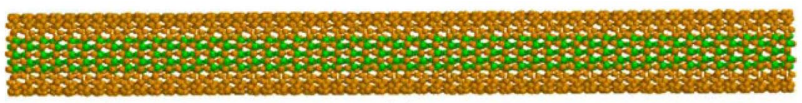

(a)

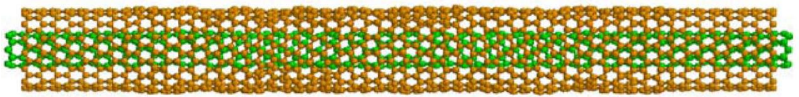

(b)

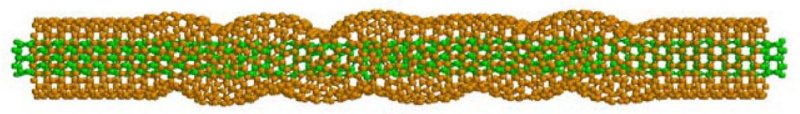

(c)

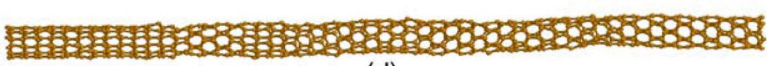

(d)

Figure 8. Snapshots of compressive deformation of uncapped $(6,0)$ $(15,0)$ nanotube at (a) $0,(b) 30$, (c) 60 ps. (d) The inner nanotube alone at $60 \mathrm{ps}$. Buckling of outer nanotube in (c) induces bending deformation in inner nanotube $(\mathrm{d})$.

$(15,0)$ uncapped double-walled nanotube at different applied strains. The inner nanotube does not experience any loading till the outer nanotube buckles (Figure 8(a and b)). It experiences bending during the post-buckling deformation of outer nanotube (Figure 8(c)). There is no increase in the compressive stress of the inner nanotube because of the nature of deformation, but there is a sharp increase in the energy per atom during bending (see Figure 9). This indicates that load is transferred to inner nanotubes during compression even when the nanotubes are uncapped (or fractured). Change in atomic positions due to Poisson's effect are small and are similar in tension and compression; however, in the case of compression severe local deformation changes the atomic positions of outer tube significantly and helps in load transfer to the inner nanotube. In tension, the change in lateral position of outer nanotube atoms is entirely due to Poisson's effect hence the load transfer to the inner nanotube is negligible.

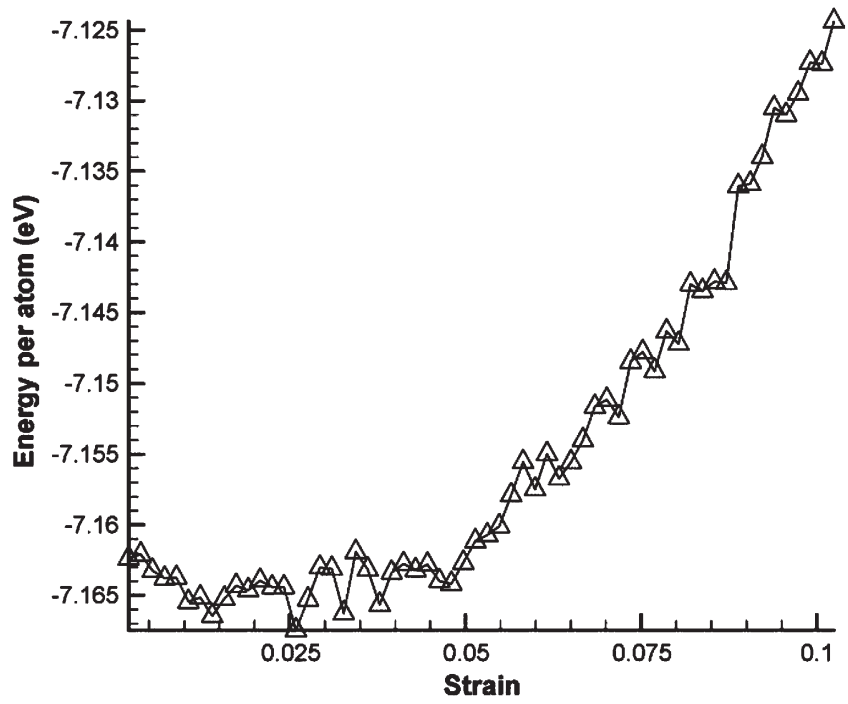

Figure 9. Energy per atom experienced by the inner tube in $(6,0)(15$, $0)$ double walled nanotube. Strain is the strain applied on the outer nanotube. Note that there is an increase in energy in $(6,0)$ nanotube after the outer nanotube buckles. 
Table 1. Stiffness values for multiwalled nanotubes in tension and compression

\begin{tabular}{lrr}
\hline Load type & $(6,0)(15,0)$ & $(6,0)(15,0)$ \\
& capped (GPa) & uncapped (GPa) \\
\hline Tension (both CNTs) & 1185 & 1094 \\
Tension (outer CNT) & 888 & 800 \\
Compression (both CNTs) & 1562 & 1590 \\
Compression (outer CNT) & 1563 & 1103 \\
\hline
\end{tabular}

Table 1 lists the elastic modulus for capped and uncapped $(6,0)(15,0)$ double walled CNT both in tension and compression. In general the compressive stiffness of CNTs is higher than the tensile stiffness. In capped nanotubes especially, stiffness is much higher in compression than in tension because the inner tube participates in deformation. In uncapped nanotubes the stiffness in compression is lower when the outer nanotube alone is loaded, however, simulations suggest that inner nanotube participates in deformation after the buckling of outer nanotube.

Continuum based shell and beam models have been applied with reasonable success to CNTs [32, 33]. Based on classical shell theory [34] we can express the critical stress for single walled nanotube as:

$$
\begin{aligned}
\sigma^{\mathrm{cr}}= & \frac{P^{\mathrm{cr}}}{2 \pi R h}=\frac{D \pi}{2 R h m^{2} L^{2}}\left(m^{2}+\left(\frac{n L}{\pi R}\right)^{2}\right) \\
& +\frac{m^{2} L^{2} E}{2 \pi^{3} R^{3}\left(m^{2}+(n L / \pi R)^{2}\right)}
\end{aligned}
$$

here $P^{\mathrm{cr}}$ is the critical load, $D$ the bending stiffness of CNT; $L, R$ and $h$ the length, radius and thickness of CNT, respectively, $E$ the Young's modulus of material and $m$ and $n$ are integers. Critical stress at the onset of buckling is obtained by minimizing the right hand side of the above equation with respect to integers $m$ and $n$. Physically they represent the buckling mode, i.e. the number of waves nanotube subdivides at buckling. Ru [17] has extended this model to multiwall nanotube with the two tubes interacting based on van der Waals interaction. According to this model an additional term incorporating the pressure due to van der Waals interaction is added to the Equation (4) above [17]. The internal pressure exerted by the van der Waals interaction is expected to reduce the critical axial strain for buckling based on this model. It can be observed that when the inner nanotube is allowed to exert pressure on the outer nanotube (i.e. when the outer nanotube alone is loaded) the critical axial strain for buckling of outer nanotube is reduced (see Figure 6 and Figure 7). This suggests a qualitative agreement between continuum based theory and discreet atomic modeling. We now proceed to study the effect of chemical bonds between walls of nanotubes on the interfacial interaction.

\section{Effect of inter-nanotube chemical bonding}

It is clear from earlier sections that stiffness and strength of MWCNTs decrease if the inner nanotube is not loaded. A natural question arises, if we can find methods to facilitate the load transfer, for example through chemical bonding.

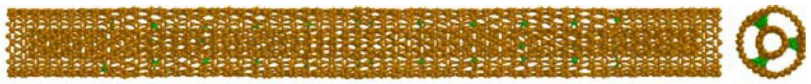

(a)

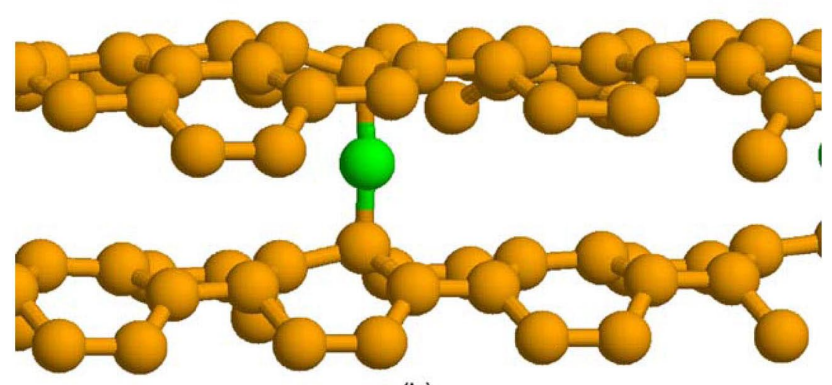

(b)

Figure 10. (a) Schematic showing interstitial atoms in multiwall nanotubes. (b) The alteration in the local structure of nanotubes near the extra carbon atoms.

Chemical bonding between the walls of carbon nanotubes has been found to be formed during irradiation of carbon nanotubes. Experimental observations of irradiation of bundles of single walled nanotube and multiwalled nanotubes indicate formation of Wigner's defect (i.e. vacancy and adjacent atom joining the walls of CNTs) as well as interstitial defects [20,21]. Figure 10 schematically shows the interstitial atoms between the walls of a double walled carbon nanotube. We have earlier observed that chemical bonding between fiber and matrix in carbon nanotube based composites greatly enhances the interfacial bonding and the overall strength of the composite [10]. Based on these results, it is to be expected that chemical interaction between the walls of multiwall carbon nanotube will enhance the inter-tube load transfer and result in stronger and stiffer multiwall nanotubes.

The effect of inter-wall chemical interaction is modeled by incorporating interstitial carbon atoms between the walls of $(6,0),(15,0)$ carbon nanotubes. About 36 carbon atoms are placed at random locations between the nanotubes of $118 \mathrm{~A}$ length. The carbon nanotubes with these interstitial defects are equilibrated by energy minimization and then subjected to tensile and compressive loading similar to previous analyses. The equilibrium structure near the defect is as shown in Figure 10(b). It can be noted that the local SP2 hybridization is disturbed by the presence of this interstitial atom.

The compressive response of the $(6,0)(15,0)$ CNT with interstitial atoms is shown in Figure 11. Plot (a) shows the virial stress versus strain when there are no interstitial atoms and both the carbon nanotubes are loaded. This corresponds to ideal load transfer between the nanotubes since both the nanotubes are simultaneously loaded. For plot (b) only the outer CNT is loaded however, the load is transmitted to inner nanotubes through interstitial atoms. It can be observed that the virial stress-strain plot in this case is closer to the ideal case in plot (a) than plot (c). Plot (c) here corresponds to the case when only the outer nanotube is loaded and there are no interstitial atoms present. In addition, if we consider the local stress of only the inner nanotube, it can be observed that the inner nanotube experiences higher stresses than for the case without interstitial atoms. Plot (d) corresponds to the stress of inner nanotube with interstitial atoms and (e) without interstitial atoms. 


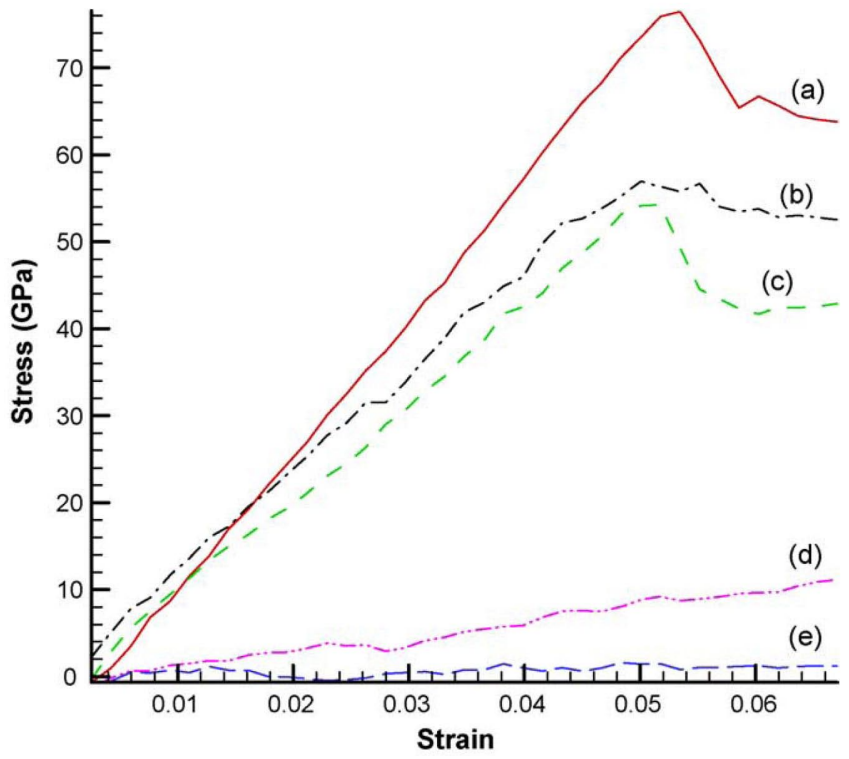

Figure 11. Compressive response of $(6,0)(15,0)$ nanotube with and without chemical bonding between the walls of nanotubes. Plots (a) both nanotubes loaded and no interstitial atoms, (b) only outer nanotube loaded with interstitial atoms, (c) only outer nanotube loaded without interstitial atoms, (d) local stress-strain of inner nanotube with interstitial atoms and (e) without interstitial atoms.

The tensile response of double-walled carbon nanotube in the presence of interstitial atoms is similar to that of the compressive response. We find that the local tensile stress experienced by the inner nanotube is enhanced considerably by the presence of interstitial carbon atoms as shown in Figure 12. Here, plot (d) shows the stress experienced by the inner nanotube with interstitial carbon atoms and plot (e) shows the stress with out interstitial carbon atoms. Further the overall stiffness of the double walled CNT is increased when interstitial atoms are present (compare plots (b and c) of Figure 12).

Simulations suggest that the excellent mechanical properties of multiwall carbon nanotubes can be exploited in a more efficient manner if composites or other structural applications are designed to be loaded in compression. In addition, the inter-wall load transfer and hence the overall properties of multiwall nanotubes can be improved if the nanotubes are pre-treated to create chemical bonds between the walls of nanotubes.

\section{Summary}

Load transfer between the walls of multiwall carbon nanotube has been studied using molecular dynamics simulations. We find that loading the outer wall of multiwall nanotube does not effectively transfer the load to inner nanotubes. It is possible to enhance the overall load carrying capacity of MWCNTs by chemically linking the walls through interstitial atoms. The salient observations of this paper are:

- Load transfer between nanotubes in multiwall nanotubes is extremely low in tension.

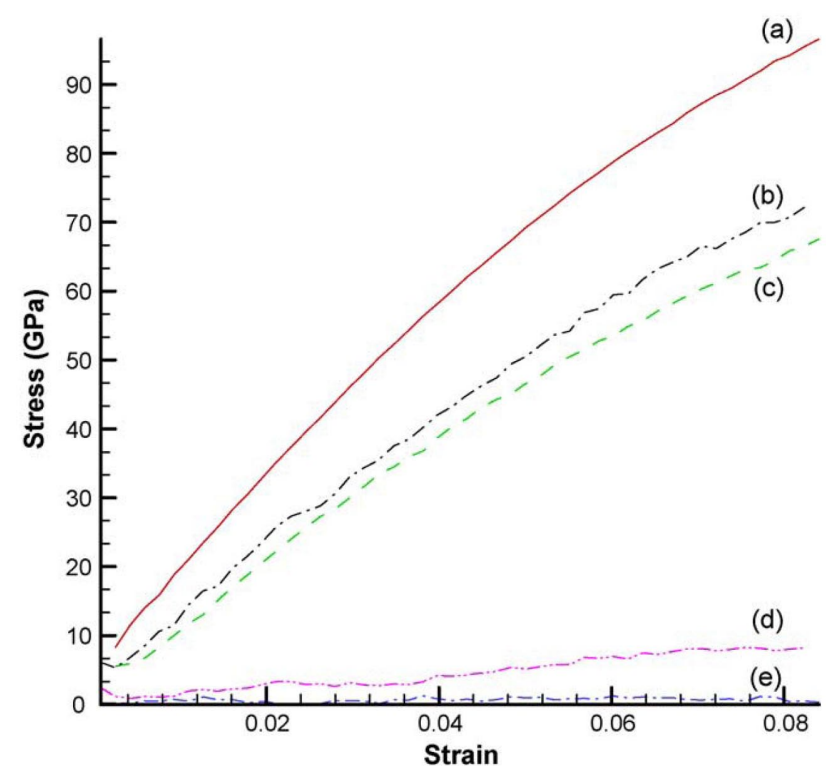

Figure 12. Tensile response of $(6,0)(15,0)$ nanotube with and without chemical bonding between the walls of nanotubes. Plots (a) both nanotubes loaded and no interstitial atoms, (b) only outer nanotube loaded with interstitial atoms, (c) only outer nanotube loaded without interstitial atoms, (d) local stress-strain of inner nanotube with interstitial atoms and (e) without interstitial atoms.

- The load transfer between the walls of capped MWCNT is much better in compression than in tension.

- In uncapped MWCNTs the inner nanotube deforms in bending during the post buckling stage of outer nanotube.

- Presence of interstitial atoms between the walls of multiwall nanotubes improves the load transfer in both tension and compression.

\section{Acknowledgments}

Financial support from ARO (Dr. Bruce La Mattina) and AFOSR (Dr. Les Lee) is gratefully acknowledged.

\section{References}

[1] M. M. J. Treacy, T. W. Ebbesen and J. M. Gibbson, Nature 381 (1996), p. 678.

[2] D. H. Robertson, D. W. Brenner and J. W. Mintmire, Phys. Rev. B 45 (1992), p. 12592.

[3] Engineered Materials Handbook, vol. 1, Composites, ASM International, 1987.

[4] B. G. Demczyk, Y. M. Wang, J. Cumings, M. Hetman, W. Han, A. Zettl and R. O. Ritchie, Mater. Sci. Eng. A 334 (2002), p. 173.

[5] X. Zhou, Eungsoo Shin, K. W. Wang and C. E. Bakis, Compos. Sci. Technol. 64 (2004), p. 2425.

[6] S. Namilae, N. Chandra, Compos. Sci. Technol. (2006), in press.

[7] Q. H. Wang, A. A. Setlur, J. M. Lauerhaas, J. Y. Dai, E. W. Seelig and R. P. H. Chang, Appl. Phys. Lett. 72 (1998), p. 2912.

[8] E. T. Thostenson, C. Y. Li and T. W. Chou, Compos. Sci. Technol. 65 (2005), p. 491. 
[9] R. B. Little, J. Clust. Sci. 14 (2001), p. 135.

[10] S. Namilae and N. Chandra, ASME J. Eng. Mater. Technol. 127 (2005), p. 222.

[11] J. Cumings and A. Zettl, Science 289 (2000), p. 602.

[12] A. N. Kolmogorov and V. H. Crespi, Phys. Rev. Lett. 85 (2000), p. 4727.

[13] J. L. Rivera, C. Mccabe and P. T. Cummings, Nano Lett. 3 (2003), p. 1001.

[14] S. Akita and Y. Nakayama, Jpn. J. Appl. Phys. 42 (2003), p. 3933.

[15] L. S. Schadler, S. C. Giannaris and P. M. Ajayan, Appl. Phys. Lett. 73 (1998), p. 3842.

[16] M. F. Yu, O. Lourie, M. J. Dyer, K. Moloni, T. F. Kelly and R. S. Ruoff, Science 287 (2000), p. 637.

[17] C. Q. Ru, J. Appl. Phys. 87 (2000), p. 7227.

[18] Z. Xia and W. A. Curtin, Phys. Rev. B 69 (2004), p. 233408.

[19] S. Namilae, N. Chandra and C. Shet, Chem. Phys. Lett. 387 (2004), p. 247.

[20] F. Banhart, Rep. Prog. Phys. 62 (1999), p. 1181.

[21] A. V. Krasheninnikov and K. Nordlund, Nucl. Instrum. Methods Phys. Res. B 216 (2004), p. 355.

[22] D. W. Brenner, Phys. Rev. B 42 (1991), p. 9458.
[23] D. W. Brenner, O. A. Shenderova, J. A. Harrison, S. J. Stuart, B. Ni and S. B. Sinnott, J. Phys. Condensed Matt. 14 (2002), p. 783.

[24] N. Chandra, S. Namilae and C. Shet, Phys. Rev. B 69 (2004), p. 094101.

[25] M. P. Allen and W. J. Tildesley, Computer Simulation of Liquids, Oxford University Press, Oxford (1989).

[26] Z. S. Basinski, M. S. Duesberry and R. Taylor, Can. J. Phys. 49 (1971), p. 2160.

[27] J. F. Lutsko, J. Appl. Phys. 64 (1988), p. 1152.

[28] J. Cormier, J. M. Rickman and T. J. Delph, J. Appl. Phys. 89 (2001), p. 99.

[29] A. Sears and R. C. Batra, Phys. Rev. B 69 (2004), p. 235406.

[30] E. W. Wong, P. E. Sheehan and C. M. Lieber, Science 277 (1997), p. 1971.

[31] O. Lourie, D. M. Cox and H. D. Wagner, Phys. Rev. Lett. 81 (1998), p. 1638.

[32] B. I. Yakobson, C. J. Brabec and J. Bernholc, Phys. Rev. Lett. 76 (1996), p. 2511.

[33] S. Govindjee and J. L. Sackman, Solid State Commun. 110 (1999), p. 227.

[34] S. P. Timoshenko and J. M. Gere, Theory of Elastic Stability, McGraw-Hill, New York (1961). 